\section{EVALUATING THE IMPLEMENTATION OF A SPORT INJURY PREVENTION PROGRAM IN A SCHOOL SETTING}

1,2 Sarah A Richmond, 'Oluwatoyosi Owoeye, 'Alberto Nettel-Aguirre, ${ }^{2}$ Alison Macpherson, ${ }^{1}$ Carla van den Berg, ${ }^{1}$ Brent Hagel, ${ }^{3}$ Evert Verhagen, ${ }^{4}$ Caroline Finch, ${ }^{1}$ Carolyn Emery. ${ }^{1}$ University of Calgary, Canada; ${ }^{2}$ York University, Canada; ${ }^{3}$ VU University Medical Centre, Netherlands: ${ }^{4}$ Monash University, Australia

\subsection{6/injuryprev-2016-042156.915}

Background Sport injuries are a significant burden, and while there are studies demonstrating the effectiveness of injury prevention programs, there is less guidance on how they are adopted and implemented successfully. The aim of this project was to conduct a process and formative evaluation of an injury prevention program implemented in a school setting.

Methods This study proposes two frameworks [RE-AIM and the Consolidated Framework for Implementation Research (CFIR)] to evaluate the implementation of a neuromuscular training program (iSPRINT) to reduce sport injury and improve healthrelated measures in a junior high school population. The five dimensions of the RE-AIM and CIFR frameworks will be used.

Results A total of 245/320 students were willing to participate in the program (68\% reach). There was a lower risk of injury in intervention schools $(\mathrm{n}=2)$ compared to controls $(\mathrm{n}=2)$ $[\mathrm{RR}=0.52$ (95\% CI: $0.33-0.81)] .76 \%$ of students and $83 \%$ of teachers reported positive attitudes towards the program, $60 \%$ of students reported they believed the program could reduce the risk of injury, and $74 \%$ reported the program could improve fitness. $14 \%$ of the schools approached agreed to participate in the program (adoption). For students, the most frequently reported reason for participating was the belief that the program would reduce injury and increase fitness. Factors related to successful implementation of the program in schools included clear explanation and demonstration of the program, and barriers included difficulty in executing certain program components. Finally, $88 \%$ of teachers reported interest in maintaining the program. Ongoing formative evaluation will be collected via focus groups using CFIR constructs (2015-2016).

Conclusions Teachers were able and keen to continue to use the iSPRINT program. We will provide further discussion on the dimensions of REAIM, and report the specific constructs that facilitated and impeded implementation of the iSPRINT program.

\section{A FIVE-YEAR TIME TREND ANALYSIS OF ROAD TRAFFIC INJURIES [RTIS] AND DEATHS AMONG INFANTS AND TODDLERS IN QATAR}

${ }^{1}$ Rafael Consunij, ${ }^{2}$ Amber Mehmood, ${ }^{1}$ Furqan Irfan, ${ }^{1}$ Rania Abdelhamid, ${ }^{1}$ Ruben Peralta, ${ }^{1}$ Ayman El-Menyar, ${ }^{2}$ Katharine A Allen, ${ }^{1,2}$ Shahnaz Malik, ${ }^{1}$ Hassan Al-Thani, ${ }^{2}$ Adnan Hyder. ${ }^{1}$ Hamad Medical Corporation, Qatar; ${ }^{2} J o h n s$ Hopkins Bloomberg School of Public Health, U. S.A

\subsection{6/injuryprev-2016-042156.916}

Background Road Traffic Injuries (RTIs) are the leading cause of death in Qatar, ${ }^{1}$ but the epidemiology of these injuries in the infant $(0-1$ years) and toddler ( $2-4$ years) [IAT] population has not been reported. This study aimed to document and analyse the epidemiology of RTIs in IATs of Qatar and make recommendations for targeted and age-specific recommendations to improve road safety for this population.

Methods A retrospective analysis of data on child RTIs and RTI deaths admitted to the Hamad Medical Corporation [HMC]
Trauma Centre or Mortuary in Doha, Qatar, from 2008-2014, was conducted. Temporal trends in the nature of RTI's and RTI deaths, road user types and mortality rates were calculated and analysed for the years that age-group population size was available.

Results There were 189 severe RTIs and 15 RTI deaths during the study period. Males made up $80 \%$ of the injured and $60 \%$ of fatalities. The average age of the injured was 3 years and for fatalities was 2.8 years. Pedestrians [53\%] and unrestrained passengers [43\%] made up the majority of the injured. There have been steady declines in severe RTI and RTI death rates from 2008 to 2012 [ 25.9 to 22.2 RTIs per 100,000 and 9.0 to 4.0 RTI deaths per 100,000], but these rates are still two times higher than those for IAT in other high-income countries [HICs] like the United States and Germany. ${ }^{2}$

Conclusions RTIs and RTI death rates in IAT in Qatar have been declining but proven programs for improved safety of child pedestrians and passengers must be implemented if it is to approximate those in other HICs. This includes programs around child restraint use and improving pedestrian environments and practices/supervision for IAT.

\section{REFERENCES}

1 Consunii RJ, Peralta RR, Al-Thani $\mathrm{H}$, et al. The implications of the relative risk for road mortality on road safety programmes in Qatar. Inj Prev Published Online First: [29 January 2014] doi:10.1136/injuryprev-2013-040939.

2 World Health OrganisationOrganization. Global status report on road safety 2015 supporting a decade of action: summary 2015.

\section{PROACTIVE AND REACTIVE PARENT-CHILD CONVERSATIONS ABOUT INJURY}

Elizabeth O'Neal, Jodie Plumert. University of Iowa, USA

\subsection{6/injuryprev-2016-042156.917}

Background Parents are uniquely positioned to reduce unintentional injury risk in children. Early in childhood this takes the form of direct supervision, yet as children gain independence parents must devise new ways of keeping their children safe. Parent-child conversations about safety may help children to internalise safety values so that they are able to independently regulate their own behaviour. This paper reports studies that examine how these conversations unfold in both proactive (before an injury occurs) and reactive (after an injury has occurred) situations.

Methods We studied proactive conversations by asking mothers and their children (8- to 10-years-old) to rate and discuss the safety of 12 photographs depicting children engaged in various physical activities. To examine reactive conversations, we examined interviews with parents about the strategies they used when discussing how an injury requiring a trip to the emergency department could have been prevented.

Results In proactive conversations, parents referenced the potential outcomes of the activity and the dangerous features of the situation. In reactive conversations, parents relied on 4 main strategies: 1) providing an alternative strategy for future use, 2) telling children to be more careful in the future, 3) telling children not to engage in the activity again, and 4) explaining why the activity was dangerous. Parents varied their use of these strategies according to children's age, gender, and the circumstances surrounding the injury.

Conclusions Parents promote the internalisation of safety values by pointing out dangerous features and outcomes of a situation, as well as by explaining why certain behaviours are dangerous. In 